\title{
Blur Detection for Digital Images Using Wavelet Transform*
}

\author{
Hanghang Tong \\ Department of Automation \\ Tsinghua University \\ Beijing 100084, China \\ walkstar98@mails.tsinghua.edu.cn \{mjli,hjzhang\}@microsoft.com \\ Mingjing Li, Hongjiang Zhang \\ Microsoft Research Asia \\ 49 Zhichun Road \\ Beijing 100080, China
}

\author{
Changshui Zhang \\ Department of Automation \\ Tsinghua University \\ Beijing 100084, China \\ zcs@tsinghua.edu.cn
}

\begin{abstract}
With the prevalence of digital cameras, the number of digital images increases quickly, which raises the demand for image quality assessment in terms of blur. Based on the edge type and sharpness analysis using Harr wavelet transform, a new blur detection scheme is proposed in this paper, which can determine whether an image is blurred or not and to what extent an image is blurred. Experimental results demonstrate the effectiveness of the proposed scheme.
\end{abstract}

\section{Introduction}

With the popularization of digital cameras, many home users have collected more and more digital photos. However, due to the lack of expertise, some of these images are of poor quality. One of the key factors that lead to quality degradation is blur. To help home users restore those photos or simply discard them, automatic blur detection is highly desirable. That is to judge whether or not a given image is blurred and to determine to what extent the image is blurred.

As far as we know, few research efforts have been made to judge whether or not a given image is blurred up till now. However, based on the explicit or implicit assumption that the given image is blurred, some existing methods can be used to determine the blur extent, which can be categorized into indirect and direct methods.

Linear blur can be modeled as [1]

$$
G=H * F+N
$$

where matrices $G, F$ and $N$ represent, respectively, the noisy blurred image, the original image and the noise, while the matrix $H$ represents the blur function. Indirect methods rely on the reconstruction of blur function $H$ when it is unknown, which are essentially blur identification and blur estimation. In the past few decades, there has been a large amount of related research. For example, Panchapakesan et al in [4] proposed a blur identification approach using vector quantizer encoder distortion; Pavlovic et al in [5] resort to maximum likelihood to identify blur function; Rooms et al in [6] proposed to estimate the out-offocus blur in wavelet domain by examining the sharpness of the sharpest edges. Although blur function can be used as an indictor of blur extent, the computational cost is very high.

Compared with the large amount of research focusing on indirect methods, it seems that not much attention has been paid to direct methods. Direct methods determine blur extent by examining some discriminative features. One of such features is edge. When blur occurs, both the edge type and its sharpness will change. By examining the absence of alternating component (AC) coefficients which indicate the edge sharpness, the authors of [3] proposed using DCT information to qualitatively characterize blur extent. However, it will lose its efficiency for over-illuminated or dark images and those with uniform background.

In this paper, we propose a new blur detection scheme using Harr wavelet transform, which belongs to direct methods. It can not only judge whether or not a given image is blurred, which is based on edge type analysis, but also determine to what extent the given image is blurred, which is based on edge sharpness analysis. The proposed scheme takes advantage of the ability of Harr wavelet transform in both discriminating different types of edges and recovering sharpness from the blurred version. It is effective for both Out-of-focus blur and Linear-motion blur. Furthermore, its effectiveness will not be affected by the uniform background in images. The proposed scheme is fast since it does not have to reconstruct the

* This work was performed at Microsoft Research Asia. 
blur function $H$.

The rest of the paper is organized as follows: In section 2, we present our scheme in detail; experimental results are given in section 3; finally, we conclude the paper in section 4 .

\section{Blur detection scheme}

Different edges are generally classified into three types: namely, Dirac-Structure, Step-Structure and Roof-Structure $[8,9,10]$. In this paper, we further classify Step-Structure into Astep-Structure and GstepStructure according to whether the change of intensity is gradual or not. A graphical description of different types of edges is given in Fig. 1. Note that for GstepStructure and Roof-Structure edge, there is a parameter $\alpha \quad(0<\alpha<\pi / 2)$ indicating the sharpness of the edge: the larger $\alpha$ is, the sharper the edge is.

The basic idea of our scheme is as follows: In general, most natural images contain all types of edges more or less, and most Gstep-Structure and RoofStructure are sharp enough. When blur occurs, no matter whether it is caused by Out-of-focus or Linearmotion, both Dirac-Structure and Astep-Structure will disappear. What is more, both Gstep-Structure and Roof-Structure tend to lose their sharpness. Our scheme judges whether a given image is blurred according to whether it has Dirac-Structure or AstepStructure, and uses the percentage of Gstep-Structure and Roof-Structure which are more likely to be in a blurred image to determine the blur extent. The whole structure of our scheme is shown in Fig. 2.

In this section, we will first analyze blur effect on different types of edges in subsection 2.1; then Harr wavelet transform for edge detection will be given in subsection 2.2 and its ability in both discriminating blur types and recovering sharpness from the blurred version will be carefully examined and summarized; finally, subsection 2.3 will give the detail of the proposed scheme.

\subsection{Blur effect on different edges}

Since there is not much noise in images acquired from digital cameras, the noise item $N$ in (1) can be neglected. The main blur functions $H$ are Out-offocus and Linear-motion. In both cases, the convolution operation will change the edge property. An empirical comparison between the original edge and its blurred version is listed in table1.

Note that in the blurred version, there is no DiracStructure or Astep-Structure. Furthermore, both Gstep-Structure and Roof-Structure tend to lose their sharpness.

Table1. Blur effect on different types of edges

\begin{tabular}{|c|c|c|}
\hline Original & After Blur & Change of $\alpha$ \\
\hline Dirac-Structure & Roof-Structure & \\
\hline Astep-Structure & Gstep-Structure & \\
\hline Gstep-Structure & Gstep-Structure & Smaller \\
\hline Roof-Structure & Roof-Structure & Smaller \\
\hline
\end{tabular}

\subsection{Edge type and sharpness detection}

Wavelet Transform is well known for its multiresolution analysis ability. Based on the important fact that local maxima of a wavelet transform detect the location of irregular structures which is proved in [2], the authors of $[9,10]$ further use modulus-angleseparated wavelet (MASW) to detect Dirac-Structure and Step-Structure edge respectively. However, MASW is very time consuming. So in this paper, we use Harr wavelet transform (HWT) instead, which is widely used $[7,8]$.

The detailed algorithm to detect edge using Harr wavelet transform is listed below:

\section{Algorithm 1: HWT for edge detection}

Step1: Perform Harr wavelet transform to the original image and the decomposition level is 3 . The result is a hierarchical pyramid-like structure (Fig. 3);

Step2: Construct the edge map in each scale:

$$
\operatorname{Emap}_{i}(k, l)=\sqrt{L H_{i}^{2}+H L_{i}^{2}+H H_{i}^{2}}(i=1,2,3)
$$

Step3: Partition the edge maps and find local maxima in each window. The window size in the highest scale is $2 \times 2$, the next coarser scale is $4 \times 4$, and the coarsest one is $8 \times 8$. The result is denoted as $E \max _{i}(i=1,2,3)$.

In algorithm 1, $E \max _{i}$ represents the intensity of the edge: the larger $E \max _{i}$ is, the more intense the edge is. For a given threshold, if $E \max _{i}(k, l)>$ threshold,$(k, l)$ is labeled an edge point in the corresponding scale; otherwise it is labeled a non-edge point.

We perform algorithm 1 on different types of edges and find that the effect is quite different. The relative intensity in different scales of different types is summarized in table2.

Table2. Effect of HWT on different types of edges

\begin{tabular}{|l|l|l|l|}
\hline & Emax1 & Emax2 & Emax3 \\
\hline Dirac-Structure & Highest & Middle & Lowest \\
\hline Astep-Structure & Highest & Middle & Lowest \\
\hline Gstep-Structure & Lowest & Middle & Highest \\
\hline \multirow{2}{*}{ Roof-Structure } & Lowest & Middle & Highest \\
\cline { 2 - 4 } & Lowest & Highest & Middle \\
\hline
\end{tabular}


Another important property of Harr wavelet transform is its ability to recover the sharpness of the blurred edge: when observed in small scale, the blurred Roof-Structure and Gstep-Structure will become thinner and thus recover their sharpness.

The above two important properties of Harr wavelet transform lead to the following five rules:

Rule1: if $\quad E \max _{1}(k, l)>$ threshold or $E \max _{2}(k, l)>$ threshold or $E \max _{3}(k, l)>$ threshold , $(k, l)$ is an edge point.

Rule2: For any edge point $(k, l)$, if $E \max _{1}(k, l)>E \max _{2}(k, l)>E \max _{3}(k, l) \quad, \quad(k, l) \quad$ is

Dirac-Structure or Astep-Structure.

Rule3: For any edge point $(k, l)$, if $E \max _{1}(k, l)<E \max _{2}(k, l)<E \max _{3}(k, l) \quad, \quad(k, l) \quad$ is

Roof-Structure or Gstep-Structure.

Rule4: For any edge point $(k, l)$, if $E \max _{2}(k, l)>E \max _{1}(k, l)$ and

$E \max _{2}(k, l)>E \max _{3}(k, l),(k, l)$ is Roof-Structure.

Rule5: For any Gstep-Structure or Roof-Structure edge point $(k, l)$, if $E \max _{1}(k, l)<$ threshold,$(k, l)$ is more likely to be in a blurred image.

\subsection{Proposed blur detection scheme}

Based on the five rules obtained in the previous subsection, the detail of the scheme is given below:

\section{Algorithm2: blur detection scheme.}

Step1: Perform algorithm 1 on the given image;

Step2: Use Rule 1 to find all edge points. Let $N_{\text {edge }}$ be the total number of them;

Step3: Use Rule 2 to find all Dirac-Structure and Astep-Structure edge points. Let $N_{d a}$ be the total number of them;

Step4: Use Rule 3 and Rule 4 to find all RoofStructure and Gstep-Structure edge points. Let $N_{r g}$ be the total number of them;

Step5: Use Rule 5 to find all Roof-Structure and Gstep-Structure edge points that have lost their sharpness. Let $N_{b r g}$ be the total number of them;

Step6: Calculate the ratio of Dirac-Structure and Astep-Structure to all the edges: $\operatorname{Per}=\frac{N_{d a}}{N_{\text {edge }}}$, if Per $>$ MinZero, judge that the image is un-blurred and vice versa, where MinZero is a positive parameter close to zero;

Step7: Calculate how many Roof-Structure and GstepStructure edges are blurred: BlurExtent $=\frac{N_{b r g}}{N_{r g}}$. Output BlurExtent as blur confident coefficient for the image.

\section{Experimental results}

In this section, we will show some experimental results. There are two parameters needed to be set: threshold and MinZero. Since human vision systems are not sensible to intensity below intensity 30, we choose 35 as threshold. In ideal situation, MinZero should be set to zero. In practice, it can be chosen as a positive real number that is very close to zero. In our experiment, we set it to be 0.05 and find out that the final accuracy only changes a little when MinZero varies between 0.01 and 0.1 .

The un-blurred images used in the experiment are taken from Corel database, and the blurred ones are simulated using equation (1) without the noise term. The experimental results are listed in table3. An example demonstrating the effectiveness of our scheme for determining blur extent is shown in Fig. 4.

Table3. Experimental result of proposed scheme

\begin{tabular}{|c|c|c|}
\hline Test Image Set & Number & Accuracy (\%) \\
\hline Un-blurred & 1398 & 98.21 \\
\hline Motion Blur & 479 & 98.33 \\
\hline Out-of-focus Blur & 458 & 100 \\
\hline Total & 2355 & 98.60 \\
\hline
\end{tabular}

Harr wavelet transform accounts for most of the processing time. In practice, the time needed is mainly determined by the image size. For a $256 \times 384$ image, the consuming time is about one second (Intel(R) Celeron(TM) 1300MHz, 256M RAM).

\section{Conclusion}

In this paper, we have proposed a new blur detection scheme for images taken by digital cameras. The scheme makes use of the ability of Harr wavelet transform in both discriminating different types of edges and recovering sharpness from the blurred version. In general, any natural image contains all types of edges more or less. What is more, the unblurred Gstep-Structure and Roof-Structure are sharp enough. When blur occurs, 1) all Dirac-Structure and Astep-Structure will disappear; 2) both GstepStructure and Roof-Structure tend to lose their sharpness. The scheme judges whether or not an image is blurred according to whether or not it 
contains any Dirac-Structure or Astep-Structure. Blur extent is characterized by blur confident coefficient based on the percentage of Gstep-Structure and RoofStructure which are more likely to be in a blurred image. Experimental results show that the proposed scheme is effective and efficient.

\section{References}

[1] R. L. Lagendijk, Basic Method for Image Restoration and identification, Academic Press, 2000.

[2] S. Mallat and W.L. Hwang, "Singularity Detection and Processing with Wavelet," IEEE Trans. On Information Theory, March 1992, pp.617-643.

[3] X. Marichal, W.Y. Ma and H.J. Zhang, "Blur Determination in the Compressed Domain Using DCT Information," Proceedings of the IEEE ICIP'99, pp. 386-390.

[4] K. Sheppard, D.G. Marcellin, M.W. Marcellin, and B.R. Hunt, "Blur identification from vector quantizer encoder distortion," IEEE Trans. On Image Processing, March 2001, pp. 465-470.

[5] G. Pavlovic, A.M. Tekalp, "Maximum likelihood parametric blur identification based on a continuous spatial domain model," IEEE Trans. On Image Processing, October 1992, pp. 496-504.

[6] F. Rooms, and A. Pizurica, "Estimating image blur in the wavelet domain", ProRISC 2001, pp. 568-572.

[7] Y. Sun and S. T. Bow, "Fast Wavelet Transform for Color Image Compression," Proceedings of the IEEE ICIP'96, pp. 541-544.

[8] Y.Y. Tang, and L. Feng, "Multi-resolution Adaptive Wavelet Edge Detection," The 2th International Conference on Multimodal Interface, January 1999, v7-v11.

[9] Y.Y. Tang, and L. Yang, "Characterization and Detection of Edges by Lipschitz Exponents and MASW Wavelet Transform," 14th International Conference on Pattern Recognition, August 1998, pp. 1572-1574.

[10] Y.Y. Tang, L. Yang and J. Liu, "Characterization of Dirac-Structure Edges with Wavelet Transform," IEEE Trans. Systems, Man and Cybernetics, Part B, February 2000, pp.93-109.

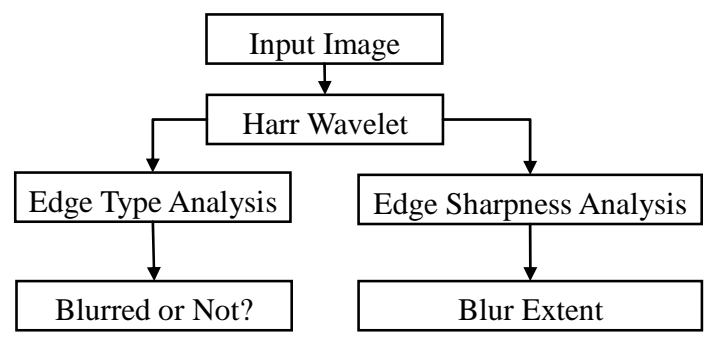

Fig. 2. Structure of the blur detection scheme

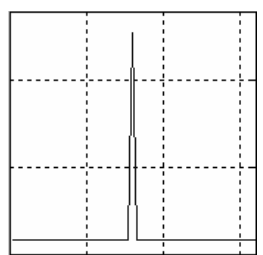

a. Dirac-Structure

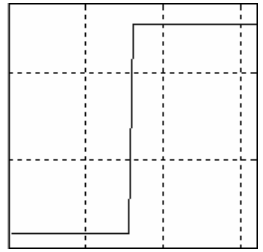

c. Astep-Structure

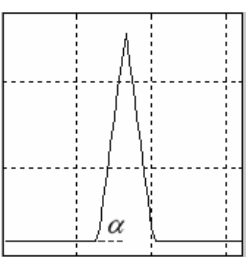

b. Roof-Structure

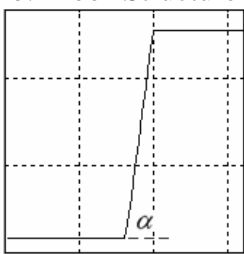

d. Gstep-Structure
Fig. 1. Graphic description of edge type

\begin{tabular}{|c|c|c|c|}
\hline $\mathrm{LL}_{3}$ & $\mathrm{HL}_{3}$ & \multirow{2}{*}{$\mathrm{HL}_{2}$} & \\
\cline { 1 - 1 } $\mathrm{LH}_{3}$ & $\mathrm{HH}_{3}$ & \\
\cline { 1 - 2 } $\mathrm{LH}_{2}$ & $\mathrm{HH}_{2}$ : Horizontal Detail & \\
\hline & & \\
$\mathrm{LH}_{1}$ : Vertical Detail & $\mathrm{HH}_{1}$ : Diagonal Detail \\
& \\
\end{tabular}

Fig. 3. Pyramid of images with related sub-bands. $\mathbf{H H}_{\mathbf{i}}$ is horizontal high-pass/vertical high-pass; $\mathbf{H L}_{\mathbf{i}}$ is horizontal high-pass/vertical low-pass; $\mathbf{L H}_{\mathbf{i}}$ is horizontal low-pass/vertical high-pass. $\quad \mathbf{L L}_{\mathbf{i}}$ is iteratively split as shown.

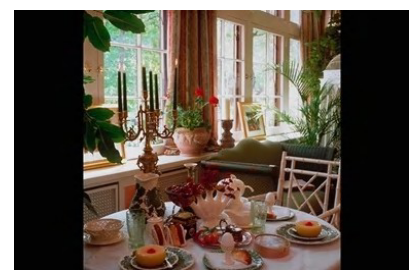

a. Original

BlurExtent $=0.0104$

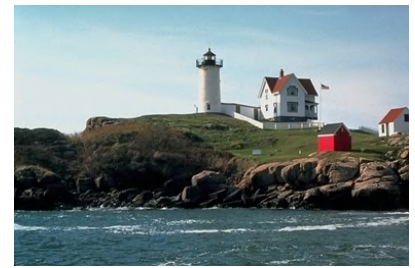

c. Original

BlurExtent $=0.0462$

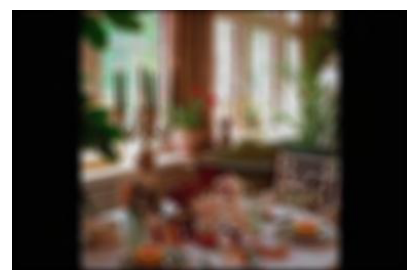

b. Out-of-focus BlurExtent $=0.4015$

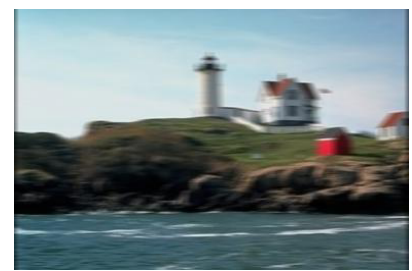

d. Linear-motion BlurExtent $=0.2095$
Fig. 4. An example of the proposed scheme to determine the blur extent 